\title{
Predictores de Mortalidad en Infecciones de Pacientes Neutropénicos Febriles Críticos.
}

\section{Predictors of Mortality in Infections of Critical Febrile Neutropenic Patients.}

\section{Karina Marín1, Henry Caballero', Víctor Figueroa1, Luis Unigarro1, Gustavo Del Pozo², Nadia Montero3, Daniel Simancas-Racines3* (D.}

*Correspondencia:

dsimancas@ute.edu.ec

Teléfono [593] 22990800

Conflicto de intereses: Los autores declaran no tener conflictos de intereses.

Fondos: Ver la página 134

Recibido: 13 Junio 2017

Aceptado: 20 Junio 2018

Publicado: 30 Agosto 2018

Membrete bibliográfico: Marín K, Caballero H, Figueroa V, Unigarro L, Del Pozo G, Montero N, Simancas-Racines D. Predictores de mortalidad en infecciones de pacientes neutropénicos febriles críticos. Rev. Oncol. Ecu 2018;28(2):128-137.

DOI: https://doi.org/10.33821/228

Copyright Marín, et al. Este artículo es distribuido bajo los términos de Creative Commons Attribution License, el cual permite el uso y redistribución citando la fuente y al autor original.
1. Unidad de Terapia Intensiva. Hospital Oncológico "Solón Espinosa Ayala" SOLCA (Quito-Ecuador)

2. Unidad de Terapia Intensiva. Hospital de Especialidades de las FFAA, HE-1, Quito-Ecuador.

3. Centro de Investigación en Salud Pública y Epidemiología Clínica (CISPEC). Facultad de Ciencias de la Salud Eugenio Espejo. Universidad Tecnológica Equinoccial. Quito-Ecuador.

\section{Resumen}

Introducción: La mortalidad relacionada a la neutropenia febril puede ser muy alta en ciertas poblaciones de alto riesgo. El objetivo del presente estudio fue identificar factores predictores tempranos de mortalidad en una cohorte de pacientes oncológicos con neutropenia febril admitidos en una unidad de cuidados intensivos (UCl).

Métodos: Estudio observacional retrospectivo en pacientes con neutropenia febril mayores de 18 años ingresados en la UCI del Hospital Oncológico Solón Espinosa Ayala de Quito. Se recogieron variables sociodemográficas, clínicas, de laboratorio y microbiológicas de los registros clínicos al ingreso. Con dichas variables se construyeron modelos predictivos de mortalidad mediante análisis de regresión logística para identificar predictores de muerte.

Resultados: Se registraron 107 pacientes con episodios de neutropenia febril, el $53.3 \%$ de los casos presentaron neutropenia severa y en un $29.9 \%$ de los casos la neutropenia duró más de 10 días. La prevalencia de bacteriemia fue del $34.6 \%$. El $34.6 \%$ de los pacientes murieron $(n=37)$, de estos el 22.4 $\%$ falleció en la UCl. Edad superior de 40 años, valores de procalcitonina superiores a $4 \mathrm{ng} / \mathrm{ml}$, puntuaciones altas en APACHE II y la necesidad de apoyo ventilatorio se asociaron con mayor riesgo de muerte en el modelo multivariado; el valor predictivo en la validación interna tuvo una precisión de $81.3 \%$; sensibilidad de $63.6 \%$; especificidad de $90.5 \%$; valor predictivo positivo de $77.8 \%$; valor predictivo negativo de $82.6 \%$; área bajo la curva de 0.87

Conclusión: Factores como la edad mayor de 40 años, procalcitonina $>4 \mathrm{ug} / \mathrm{ml}$ al ingreso, valores de APACHE II y necesidad de ventilación mecánica están asociados con mayor riesgo de muerte. 
Palabras Claves: SEPSIS, PROTEÍNAS DE FASE AGUDA, NEUTROPENIA FEBRIL, CUIDADOS CRÍTICOS.

DOI: $10.33821 / 228$

\section{Abstract}

Introduction: The mortality related to febrile neutropenia may be very high in certain populations. The aim of the present study was to identify early predictors of mortality in a cohort of oncologic patients with febrile neutropenia admitted to an intensive care unit (ICU)..

Methods: Retrospective observational study in patients with febrile neutropenia older than 18 years admitted to the intensive care unit of the "Solon Espinosa Ayala" Oncology Hospital in Quito. Sociodemographic, clinical, laboratory and microbiological variables were collected from clinical records at admission. With these variables, predictive mortality models were constructed using logistic regression analysis to identify predictors of death.

Results: There were 107 episodes of febrile neutropenia, 53.3 \% had severe neutropenia, and in 29.9 $\%$ of the cases neutropenia lasted more than 10 days. The prevalence of bacteremia was $34.6 \%$. 34.6 $\%$ of the patients died $(n=37)$, of which $22.4 \%$ died in the ICU. A higher age of 40 years, procalcitonin values higher than $4 \mathrm{ng} / \mathrm{ml}$, high APACHE II scores and the need for ventilatory support were associated with an increased risk of death in the multivariate model; The predictive value in the internal validation had an accuracy of $81.3 \%$; Sensitivity of $63.6 \%$; Specificity of $90.5 \%$; Positive predictive value of $77.8 \%$; Negative predictive value of $82.6 \%$; Area under the curve of 0.87

Conclusion: Factors such as age greater than 40 years, procalcitonin at admission $>4 \mathrm{ug} / \mathrm{ml}$, APACHE II and requirement of mechanical ventilation support are associated with a higher risk of death.

Keywords: SEPSIS, PROTEINS OF ACUTE PHASE, NEUTROPENIA FEBRIL, CRITICAL CARE.

DOI: $10.33821 / 228$

\section{Introducción}

Ya sea por la misma enfermedad neoplásica o por tratamientos citotóxicos el desarrollo de neutropenia es frecuente en pacientes oncológicos y los deja susceptibles a infecciones graves que pueden provocar cuadros de sepsis severa, choque séptico, disfunción orgánica múltiple y aumentan el riesgo de muerte [1-9].

La inmunosupresión causada por la neutropenia hace difícil reconocer con prontitud a pacientes con cuadros infecciosos en sus estadios iniciales retrasando su manejo y empeorando su pronóstico vital, por lo que la búsqueda de marcadores y factores que permitan identificar tempranamente a pacientes con mayor riesgo de estas complicaciones resulta de gran utilidad en las Unidades de Cuidados Intensivos (UCI) [10-14].

Uno de los factores más estudiados como predictor de mortalidad es la procalcitonina (PCT), un polipéptido sérico, que ha sido señalado como un biomarcador temprano de infección en pacientes inmunocompetentes [15-17] y en pacientes neutropénicos [5-8, 1822], sin embargo, su utilidad para predecir desenlaces adversos como muerte solo o en asociación con otros marcadores de gravedad como edad, comorbilidades, estado 
funcional previo, índices de severidad y el requerimiento de soporte multiorgánico, no se ha estandarizado.

El objetivo del presente estudio fue identificar factores predictores tempranos de mortalidad en una cohorte de pacientes oncológicos con neutropenia febril críticamente enfermos.

\section{Materiales y Métodos}

Se revisó una cohorte retrospectiva de pacientes. Los datos fueron tomados de los registros clínicos de pacientes mayores de 18 años con neutropenia febril, ingresados en la UCI del Hospital Oncológico Solón Espinosa Ayala (Quito-Ecuador), desde el 1 de enero del 2010 al 31 de diciembre del 2014. Se recopilaron variables demográficas, índices de severidad, soportes de fallos orgánicos y niveles de procalcitonina al ingreso.

La neutropenia febril se definió como: contaje absoluto de neutrófilos $\leq 500 \mathrm{~mm}^{3} 0<1000$ $\mathrm{mm}^{3}$ con expectativa a disminuir a $<500 \mathrm{~mm}^{3}$ en las próximas 24 horas y presencia de temperatura central $\geq 38.3^{\circ} \mathrm{C}$ por una ocasión o temperatura $\geq 38^{\circ} \mathrm{C}$ por al menos una hora. Neutropenia profunda se definió como un contaje absoluto de neutrófilos menor a 100 por $\mathrm{mm}^{3}$.

Se evaluaron variables demográficas como sexo y edad; variables clínicas tales como escores de gravedad (APACHE II y del Sequential Organ Failure Assessment (SOFA)), necesidad de ventilación mecánica, diagnóstico de choque; antecedentes de neoplasia hematológica, estancia en UCl, estancia hospitalaria; variables microbiológicas para definir bacteremia y variables de laboratorio tales como procalcitonina y albúmina. En el análisis estadístico las variables continuas se reportaron como promedios (+/- Desviación estándar), o como mediana con sus respectivos rangos intercuartílicos (RIQ), las variables categóricas como frecuencia y su respectivo porcentaje. Las comparaciones se realizaron previa pruebas de normalidad en las variables cuantitativas.

Las comparaciones para medias usaron pruebas de "t de student" o pruebas no paramétricas para aquellas que no cumplían los requisitos de normalidad. Las variables discretas se compararon usando pruebas de independencia (Chi-cuadrado) con ajustes a través de la prueba exacta de Fisher en caso de requerirse. Se construyeron modelos predictivos por regresión logística para determinar predictores independientes de muerte, la base fue separada aleatoriamente para validación interna con la herramienta "CaTools". La valoración de riesgo se reportó como OR con sus respectivos intervalos de confianza al $95 \%$. Todas las comparaciones se realizaron a dos colas y se consideró la significancia estadística con valores de $p$ menores a 0,05 . Los análisis se realizaron usando el paquete estadístico "R" (vers. 3.2.2; 2015). 


\section{Resultados}

Se registraron datos de 107 episodios de neutropenia febril consecutivos. La distribución de las características basales fue algo variable. No se encontraron diferencias en la distribución por sexo, los pacientes que fallecieron presentaron mayor edad $(P=0.04)$, de igual manera la valoración en los scores de gravedad como APACHE II y SOFA fueron mayores en este grupo $(P<0.00001)$. La presencia de neoplasia hematológica fue muy equilibrada en ambos grupos y se ubicó alrededor del $70 \%$. Los valores de procalcitonina evaluada al ingreso fue muy superior en el grupo de fallecidos con una mediana de 7.8 $\mathrm{ng} / \mathrm{ml}(P<0.01)$, ver tabla 1.

Tabla 1. Características de los pacientes neutropénicos febriles al ingreso en la Unidad de terapia intensiva de acuerdo al resultado final.

\begin{tabular}{|l|c|c|c|c|c|}
\hline \multirow{2}{*}{ Variable } & \multicolumn{2}{|c|}{$\begin{array}{c}\text { Sobrevivientes } \\
\mathrm{n}=70\end{array}$} & \multicolumn{2}{c|}{$\begin{array}{c}\text { Fallecidos } \\
\mathrm{n}=37\end{array}$} & \multirow{2}{*}{$P$} \\
\cline { 2 - 5 } & $\mathrm{n}$ & $\%$ & $\mathrm{n}$ & $\%$ & \\
\hline Género (femenino) & 32 & $(45.7 \%)$ & 21 & $(56.8 \%)$ & $\mathrm{NS}$ \\
\hline Neoplasia hematológica & 52 & $(74.3 \%)$ & 27 & $(73.0 \%)$ & $\mathrm{NS}$ \\
\hline & media & $\mathbf{q} 25-\mathrm{q} 75$ & media & $\mathrm{q} 25-\mathrm{q} 75$ & \\
\hline Edad (años) & 48 & $(30-62)$ & 57 & $(45-67)$ & $0.04 *$ \\
\hline APACHE II & 18 & $(15-21)$ & 25 & $(19-29)$ & $<0.00001 *$ \\
\hline SOFA & 7 & $(5-10)$ & 10 & $(8-12)$ & $<0.00001 *$ \\
\hline Albumina (g/dl) & 2.6 & $(2.2-3.1)$ & 2.3 & $(1.7-3.2)$ & NS \\
\hline Procalcitonina al ingreso $(\mathbf{n g} / \mathrm{ml})$ & 1.1 & $(0.5-6.4)$ & 7.8 & $(1.1-25.0)$ & $<0.01 *$ \\
\hline
\end{tabular}

Se diagnosticó neutropenia severa en 57 pacientes (53.3 \%), con un período de neutropenia superior a los 10 días en 32 pacientes $(29.9 \%$ ) y la prevalencia general de bacteriemia fue del $34.6 \%(n=37)$; estas variables no marcaron diferencia estadísticamente significativa en la mortalidad. Cincuenta y seis pacientes $(52.3 \%)$ desarrollaron choque durante su estancia en UTI.

De todos los pacientes de la muestra, 24 (22.4\%) fallecieron en la terapia intensiva y 13 pacientes adicionales fallecieron posteriormente durante su hospitalización con una tasa de mortalidad total de $34.6 \%(n=37)$ al egreso hospitalario.

Las tasas de mortalidad fueron superiores en pacientes que desarrollaron choque, con un incremento de riesgo OR: 2.6 (IC 95\%: 1.14 a 6.04 ; $P=0.03$ ) que no pudo replicarse en el modelo multivariado, ver tabla 2.

Cincuenta y nueve pacientes (51.1\%) necesitaron asistencia ventilatoria mecánica, de ellos 35 pacientes $(59.3 \%)$ fueron sometidos a ventilación invasiva. El soporte ventilatorio mecánico, una edad superior a los 40 años, valores de procalcitonina al ingreso superiores a $4 \mathrm{ng} / \mathrm{ml}$ y el puntaje en el score de APACHE II se asociaron significativamente con el riesgo de muerte en el modelo multivariado, el valor predictivo en la validación interna resultó con una precisión total $81.3 \%$; sensibilidad: $63.6 \%$; especificidad: $90.5 \%$; valor predictivo positivo (VPP): $77.8 \%$; valor predictivo negativo (VPN): $82.6 \%$; ver figura 1 y tabla 3. 
Tabla 2. Características de los pacientes neutropénicos febriles durante su evolución en la Unidad de terapia intensiva de acuerdo al resultado final.

\begin{tabular}{|c|c|c|c|c|c|}
\hline \multirow[t]{2}{*}{ Variables } & \multicolumn{2}{|c|}{$\begin{array}{l}\text { Sobrevivientes } \\
\qquad N=70\end{array}$} & \multicolumn{2}{|c|}{$\begin{array}{c}\begin{array}{c}\text { Fallecidos } \\
\mathrm{N}=37\end{array}\end{array}$} & \multirow[t]{2}{*}{$P$} \\
\hline & $\mathrm{n}$ & $\%$ & $\mathrm{n}$ & $\%$ & \\
\hline Neutropenia inferior a $100 \mathrm{~K} / \mathrm{ml}$ & 33 & (47.1\%) & 24 & $(64.9 \%)$ & NS \\
\hline Neutropenia de más de 10 días & 18 & $(25.7 \%)$ & 14 & $(37.8 \%)$ & NS \\
\hline Choque & 31 & $(44.3 \%)$ & 25 & $(67.6 \%)$ & 0.03 \\
\hline Necesidad de ventilación mecánica & 29 & $(30.5 \%)$ & 30 & $(81.1 \%)$ & $<0.0001$ \\
\hline \multirow[t]{2}{*}{ Bacteremia } & 23 & (32.9\%) & 14 & $(37.8 \%)$ & NS \\
\hline & media & $\begin{array}{l}\text { q25- } \\
\text { q75 }\end{array}$ & media & $\begin{array}{l}\text { q25- } \\
\text { q75 }\end{array}$ & $\mathrm{p}$ \\
\hline Estancia en UTI (días) & 6 & $(3-9)$ & 4 & $(2-9)$ & NS \\
\hline Estancia hospitalaria (días) & 18 & $\begin{array}{l}(11 \\
31)\end{array}$ & 11 & $(5-20)$ & $<0.001$ \\
\hline
\end{tabular}

K/ml: Células por ml; NS: No significativo a un nivel a=0.05.

Figura 1. Curva de Característica Operativa del Receptor (curva ROC)

Curva "ROC" para predicción de mortalidad

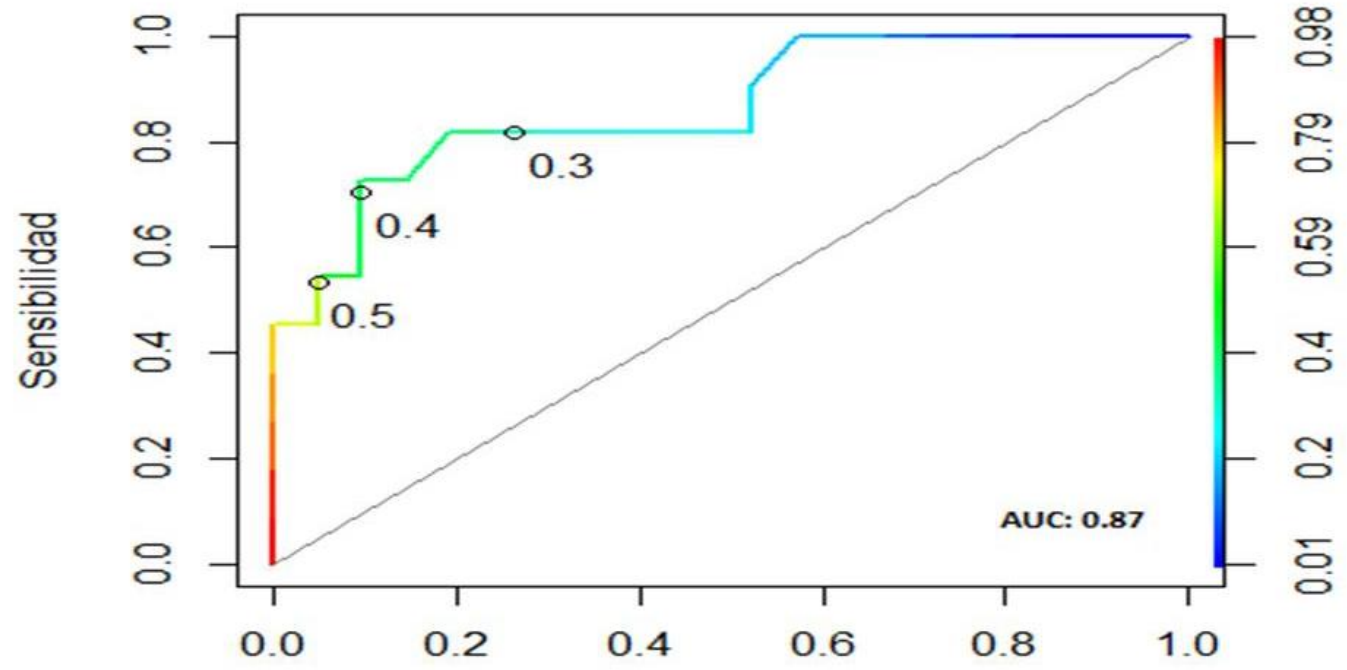

1-Especificidad

Curva ROC en el mejor modelo para predicción de mortalidad al alta hospitalaria Área bajo la curva: 0.87; Precisión total 81.3 \%; Sens: 63.6 \%; Esp: 90.5 \%; VPP: 77.8 \%; VPN: $82.6 \%$. Los factores evaluados constan en la tabla 3. Los puntos indican la probabilidad predicha por el modelo. 
Tabla 3. Factores asociados con mortalidad para pacientes neutropénicos febriles de acuerdo a los análisis multivariados (modelo de regresión logística).

\begin{tabular}{|l|c|c|c|}
\hline Factor & OR & IC 95\% & $P$ \\
\hline Ventilación mecánica* & 5.08 & 1.61 a 17.94 & $<0.01 *$ \\
\hline Edad $>$ 40 años & 6.68 & 1.93 a 28.32 & $<0.01 *$ \\
\hline Procalcitonina $>\mathbf{4 ~ u g / m l ~}$ & 3.20 & 1.11 a 9.89 & $0.04 *$ \\
\hline APACHE II & 1.11 & 1.02 a 1.21 & $0.02 *$ \\
\hline
\end{tabular}

Notas: (*) Cualquier tipo de asistencia ventilatoria mecánica; APACHE II: Score de evaluación fisiológica aguda y salud crónica (APACHE II); Intercept. 0.02.

\section{Discusión}

La mortalidad y morbilidad derivadas de las infecciones en pacientes con cáncer sigue siendo alarmante, aún más en pacientes quienes desarrollan neutropenia posterior a quimioterapia o resultante de la enfermedad neoplásica. En el presente reporte los pacientes tuvieron una mortalidad hospitalaria del $34.6 \%$ inferior a otros reportes, D. Mokart 54\% [1], M. Legrand 57 \% [2], los principales factores pronósticos independientes que encontramos fueron la mayor edad, la gravedad inicial según la evaluación del escore APACHE II, la necesidad de la ventilación mecánica y valores elevados de procalcitonina.

Es conocido que la edad avanzada es un factor de riesgo en pacientes enfermos de cáncer en estado crítico [1] y hallamos que pacientes con más de 40 años tenían mayor riesgo de morir durante su hospitalización. También el estado de gravedad al ingreso del paciente neutropénico febril está directamente relacionado con la probabilidad de morir durante la hospitalización, demostramos que una puntuación mayor de 20 en el score de gravedad APACHE II fue predictivo, otros estudios como los de Legrand [2] reportaron mayor mortalidad en aquellos pacientes con calificaciones que igualmente indican compromiso multisistémico (puntuación de 10 en el score SOFA a la admisión).

La insuficiencia respiratoria, descrita en algunos estudios como la principal causa de ingreso de los pacientes neutrópenicos febriles a la UCI también ha sido asociada como factor independiente de mortalidad. Entre el 40 al $70 \%$ de los pacientes críticos que ingresan por esta causan fallecen [18], en concordancia nosotros encontramos que los pacientes que desarrollaron insuficiencia respiratoria y que son sometidos a ventilación mecánica tienen mayor probabilidad de muerte durante la hospitalización.

El estado neutropénico es sin duda uno de los factores más importantes para el desarrollo de infecciones, bacterianas, virales y micóticas que son otro factor para desenlaces fatales. Los pacientes se hallan a menudo con molestias inespecíficas o con fiebre como único signo, esto obliga a un gran índice de sospecha para el diagnóstico temprano19. En el presente estudio encontramos que un valor superior de $4 \mathrm{ug} / \mathrm{ml}$ predice mortalidad de manera aceptable, otros estudios han llegado a conclusiones similares, valores de PCT $<5$ $\mathrm{ng} / \mathrm{dl} \mathrm{y}>5 \mathrm{ng} / \mathrm{dl}$, determinando un importante incremento en la mortalidad de $11.5 \%$ a 50 $\%$ con un OR: $7.0(P=0.04)[13]$. 
La asociación de la PCT con muerte es fuerte en este estudio, de igual manera encontramos hallazgos similares a otros estudios en la asociación de muerte con edad y gravedad valorada por APACHE II, sin embargo, la falta de inclusión de otras variables que podrían impactar en la sobrevida como presencia de comorbilidades, el estadío de la enfermedad neoplásica, su estado funcional de los pacientes podría haber influido en la capacidad predictiva de estos factores y limitado la capacidad de generalización de los presentes hallazgos.

\section{Conclusiones}

La edad mayor a 40 años, valores de procalcitonina $>4 \mathrm{ug} / \mathrm{ml}$, la valoración de APACHE II y la necesidad de ventilación mecánica, estuvieron asociados con incremento en el riesgo de muerte en pacientes neutropénicos febriles valorados en el Hospital Oncológico "Solón Espinoza Ayala" Solca de Quito, la evaluación de estos factores al ingreso puede ser de utilidad para mejorar el manejo de este tipo de pacientes.

\section{Agradecimientos}

Se reconoce a las personas que participaron indirectamente en el estudio tales como los pacientes, como personal técnico, otras en general del Hospital Solon Espinoza Ayala, Solca, Núcleo de Quito.

\section{Información adicional}

\section{Abreviaturas}

UCl: Unidad de Cuidados Intensivos.

PCT: Procalcitonina.

S: Sensibilidad.

VPP: Valor Predictivo Positivo.

VPN: Valor Predictivo Negativo.

Nota del Editor

La Revista Oncología Ecu permanece neutral con respecto a los reclamos jurisdiccionales en mapas publicados y afiliaciones institucionales.

\section{Archivos Adicionales}

Ninguno declarado por los autores.

\section{Fondos}

Los fondos de la investigación fueron propios de los autores del presente artículo. 


\section{Disponibilidad de datos y materiales}

Existe la disponibilidad de datos bajo solicitud al autor de correspondencia. No se reportan otros materiales.

\section{Contribuciones de los autores}

$\mathrm{KM}, \mathrm{HC}, \mathrm{GDP}$; DSR realizaron la idea de investigación, revisión bibliográfica, el análisis crítico del artículo. VF, LU, NM realizaron la recolección de datos, escritura del artículo. KM realizó las correcciones. DSR análisis estadístico. Todos los autores leyeron y aprobaron la versión final del artículo.

Aprobación de ética y consentimiento para participar

No aplica ya que es un estudio de revisión de historias clínicas.

\section{Consentimiento para publicación}

No aplica.

\section{Información de los autores}

Karina Marín, Médica de la Unidad de Terapia Intensiva del Hospital Oncológico "Solón Espinosa Ayala, Solca -Quito.

Henry Caballero, Médico de la Unidad de Terapia Intensiva del Hospital Oncológico "Solón Espinosa Ayala, Solca -Quito.

Víctor Figueroa, Médico de la Unidad de Terapia Intensiva del Hospital Oncológico "Solón Espinosa Ayala, Solca -Quito.

Luis Unigarro, Médico de la Unidad de Terapia Intensiva del Hospital Oncológico "Solón Espinosa Ayala, Solca -Quito.

Gustavo del Pozo, Médico de la Unidad de Terapia Intensiva del Hospital de Especialidades de las FFAA, HE-1, Quito-Ecuador.

Daniel Simancas Racines, Centro de Investigación en Salud Püblica y Epiddemiología Clínica (CISPEC). Facultad de Ciencias de la Salud Eugenio Espejo. Universidad Tecnológica Equinoccial. Quito-Ecuador. (iD) https://orcid.org/0000-0002-3641-1501 


\section{Revisiones por pares}

Acceda a la revisión de pares académicos en el siguiente enlace: https://publons.com/review/3977066

\section{Referencias}

\section{Abreviaturas en la referencias}

\section{DOI: Digital Object}

Identifier

PMID: PubMed Identifier

\section{SU: Short URL}

1. Mokart D, Darmon M, Resche-Rigon M, Lemiale V, Pene F, Mayaux J, et al. Prognosis of neutropenic patients admitted to the intensive care unit. Intensive Care Med. 2015; 41(2):296-303. DOI: $10.1007 / \mathrm{s} 00134-014-3615-y$

2. Legrand M, Max A, Peigne V, Mariotte E, Canet E, Debrumetz A, et al. Survival in neutropenic patients with severe sepsis or septic shock. Critical care medicine. 2012; 40(1), 43-49. DOI: 10.1097/CCM.0b013e31822b50c2

3. De Naurois J, Novitsky-Basso I, Gill M, Marti F, Cullen M, Roila F. Management of Febrile Neutropenia: ESMO Clinical Practice Guidelines. Annals of Oncology. 2011; 21(Suplemento 5):v252-v256. DOI: 10.1093/annonc/mdq196

4. Freifeld A, Bow E, Sepkowitz K, Boeckh M, Ito J, Mullen C, et al. Clinical practice guideline for the use of antimicrobial agents in neutropenic patients with cancer: 2010 update by the Infectious Diseases Society of America. Clinical infectious diseases. 2011;52(4): e56-e93. DOI: 10.1093/cid/cir073.

5. Neuenschwander L, Bittencourt H, Ribeiro A, Teixeira A, Teixeira M, Teixeira J, et al. Plasma levels of procalcitonin and eight additional inflammatory molecules in febrile neutropenic patients. Clinics. 2011; 66(10):1699-1705. PMID: 22012040.

6. André S, Taboulet P, Elie C, Milpied N, Nahon M, Kierzek G, et al. Febrile neutropenia in French emergency departments: results of a prospective multicentre survey. Critical care. 2010;14(2), R68:1-11. DOI: 10.1186/cc8972

7. Legrand M, Max A, Schlemmer B, Azoulay E, Gachot B. The strategy of antibiotic use in critically ill neutropenic patients. Annals of Intensive Care. 2011;1(22):1-9. DOI: 10.1186/2110-5820-1-22

8. Shin A, Yoon-Seon L. Predictive factors for poor prognosis febrile neutropenia. Current Opinion Oncol. 2012; 24(4):376-380.DOI: 10.1097/CCO.0b013e328352ead2.

9. Ford A, Marshall E. Neutropenic sepsis: a potencially life-threatening complication of chemotherapy. Clinical Medicine. 2014; 14(5):538-542. DOI: 10.7861/clinmedicine.14-5-538

10. Marin K, Caballero H, Del Pozo G, Figueroa V, Unigarro L, Basantes E, et al. Procalcitonina como predictor independiente de mortalidad en pacientes neutropénicos febriles críticos. Memorias de temas orales. XVII Congreso Panamericano de Infectología 2015: 10. SU: goo.gl/tLEu5f

11. Bonilla D, Cuervo S, Gómez J. Utilidad de la procalcitonina en pacientes adultos con neoplasias hematológicas y neutropenia febril postquimioterapia. Estado del arte. Infectio Asociación Colombiana de Infectología. 2012;16(4):223-229. SU: goo.gl/wqxGpv

12. Massaro R, Macedo R, de Castro B, Dulley F, Oliveira M, Yasuda S. Risk factor for death in hematopoietic stem cell transplantation: are biomarkers useful to foresee the prognosis in this population of patients? Infection. 2014;42:1023-1032. DOI: 10.1007/s15010-014-0685-2

13. Kitanovski L, Jazbec J, Hojker S, Derganc M. Diagnostic accuracy of lipopolysaccharide-binding protein for predictingbacteremia/clinical sepsisinchildrenwithfebrile neutropenia: comparison with interleukin6, procalcitonin, and C-reactive protein. Support Care Cancer. 2014;22:269-277. DOI: 10.1007/s00520$\underline{013-1978-1}$ 
14. Shin A, Yoon-Seon L, Kyung Soo L, Jae-Lyun L. Adding procalcitonin to the MASCC risk-index score could improve risk stratification of patients with febrile neutropenia. Support Care Cancer. 2013; 21:2303-2308. DOI: $10.1007 / \mathrm{s} 00520-013-1787-6$

15. Watanabe $Y$, Oikawa N, Hariu M, Fuke R, Seki M. Ability of procalcitonin to diagnose bacterial infection and bacteria types compared with blood culture findings. Int J Gen Med. 2016; 30(9): 325-331. DOI: $10.2147 /$ IJGM.S115277

16. Peschanski N, Chenevier-Gobeaux C, Mzabi L, Lucas R, Ouahabi S, Aquilina V, et al. Prognostic value of PCT in septic emergency patients. Ann Intensive Care. 2016 Dec;6(1):47. DOI: 10.1186/s13613-016$\underline{0146-4}$

17. Huang M, Chen C, Chien J, Wu K, Chang Y, Wu K, et al. Serum Procalcitonin and Procalcitonin Clearance as a Prognostic Biomarker in Patients with Severe Sepsis and Septic Shock. Biomed Res Int. 2016; Epub. DOI: $10.1155 / 2016 / 1758501$

18. Aimoto M, Koh H, Katayama T, Okamura H, Yoshimura T, Koh S. Diagnostic performance of serum highsensitivity procaltinonin and serum C-reactive protein tests for detecting bacterial infection in febrile neutropenia. Infection. 2014; 41(6):971-979. DOI: 10.1007/s15010-014-0657-6

19. Mokart D, Azoulay E, Schnell D, Bourmaud A, Kouatchet A, Pène F. Acute respiratory failure in neutropenic patients is associated with a high post-ICU mortality. Minerva anestesiologica. 2013; 79(10): 1156-1163. PMID: 23857442

20. Sánchez Yépez, Aznar-Oroval E, Lorente-Alegre P, García-Lozano T, Picón-Roig I, Pérez-Ballestero P, Ortiz-Munoz B. Utilización de procalcitonina y proteína $C$ reactiva como marcadores de infección en la neutropenia febril de pacientes sometidos a trasplante de progenitores hematopoyéticos. Enfermedades Infecciosas y Microbiología Clínica. 2014; 32(7), 418-423. DOI: 10.1016/j.eimc. 2013.09 .003

21. Boysen $A$, Jensen $B$, Poulsen $L$, Jensen $P$, Ladefoged $S$. Procalcitonin as a marker of infection in febrile neutropenia: A systematic review. Modern Chemotherapy. 2013; 2(01):8. DOI: 10.4236/mc.2013.21002

22. Riyaz, $M$, Manuel $R$, Joseph $N$. Importance of serum procalcitonin in febrile neutropenia. Journal of Evolution of Medical and Dental Sciences. 2014:3(29): 8012-8019. 